12th International Symposium on Cosmology and

Particle Astrophysics (CosPA 2015)

International Journal of Modern Physics: Conference Series

Vol. 43 (2016) 1660198 (7 pages)

(C) The Author(s)

DOI: $10.1142 / \mathrm{S} 2010194516601988$

\title{
Galactic Center Excess by Higgs Portal Dark Matter
}

\author{
Arindam Das and Nobuchika Okada \\ Department of Physics and Astronomy, \\ University of Alabama, Tuscaloosa, Alabama 35487, USA \\ Osamu Seto* \\ Department of Life Science and Technology, \\ Hokkai-Gakuen University, Sapporo 062-8605, Japan \\ seto@physics.umn.edu
}

Published 7 July 2016

\begin{abstract}
A $Z_{2}$ parity odd real scalar is a good candidate for dark matter in the present Universe. We consider models contain two Higgs doublet fields and one real scalar dark matter particle with mass in the range of $31-40 \mathrm{GeV}$ and annihilating into a $b \bar{b}$ pair, or with about $10 \mathrm{GeV}$ mass and annihilating into tau lepton pair. Those annihilation modes suitably explain the observed excess of the gamma-ray flux from the Galactic Center. We identify the parameter region of the model that can fit the gamma-ray excess and satisfy phenomenological constraints, such as the observed dark matter relic density and the null results of direct dark matter search experiments. Most of the parameter region is found to be within the search reach of various future experiments.
\end{abstract}

Keywords: Dark matter; cosmic gamma ray; experimental test.

PACS number: 95.35.+d

\section{Introduction}

The weakly interacting massive particle (WIMP) is a prime candidate for dark matter (DM) in the Universe and indirect search of WIMP DM through its annihilation is one of the most effective methods to detect a WIMP.

Over the past several years, many analysis have claimed an excess of gamma rays from the Galactic Center (GC) and its WIMP annihilation interpretations. ${ }^{1-4}$ It has been reported that this gamma-ray excess can be fit by about $10 \mathrm{GeV}$ mass WIMP annihilating into a pair of tau leptons, or by several tens GeV mass WIMP

*Speaker.

This is an Open Access article published by World Scientific Publishing Company. It is distributed under the terms of the Creative Commons Attribution 4.0 (CC-BY) License. Further distribution of this work is permitted, provided the original work is properly cited. 
annihilating into a $b \bar{b}$ pair. ${ }^{5-9}$ The Fermi-LAT Collaboration has released the constraints on WIMP annihilation cross section by using six years data taken from dwarf Spheroidal (dSph) galaxies, and if we take the face value for the WIMP annihilation cross section, one may find a mild tension between the Fermi-LAT constraints and a favored value for GC excess. ${ }^{10}$ However, at this moment, we are going to investigate WIMP interpretations of the GC excess, because there is an astrophysical uncertainty such as the DM density profile of galaxies or the local DM density.

One of simple models for dark matter is so-called Higgs portal DM model, where a real scalar $\phi$ with odd $Z_{2}$ parity plays a role of dark matter and annihilates into standard model (SM) particles through Higgs boson(s) exchange processes. The minimal model with $\phi$ and one $S U(2)$ doublet SM Higgs field (for an incomplete list, see, e.g., Refs. 11, 12, 13, 14) is not available for explaining this GC gamma-ray excess, because its allowed mass range is limited to be around $60 \mathrm{GeV}$ or larger than several hundreds GeV mostly by the null results of direct DM search experiments (see, for example, Refs. 15), so that the desired DM mass range $30-40 \mathrm{GeV}$ to account for the GC excess is excluded.

A desired thermal relic scalar dark matter mass is determined by the mediator particle mass in the Higgs portal DM framework. In fact, this is the reason why we have $m_{\phi} \sim 62 \mathrm{GeV} \simeq m_{h} / 2$ on the minimal model, where $h$ denotes the SM Higgs boson with the mass of $125 \mathrm{GeV}$ discovered at the Large Hadron Collider (LHC). The extension of the Higgs sector to the two Higgs doublet model (THDM) provides various possibilities.

As noted above, two annihilation modes of dark matter is favored to explain the GC excess; one is a tau lepton pair final state from about $10 \mathrm{GeV}$ mass dark matter, the other is $b \bar{b}$ final state from $30-40 \mathrm{GeV}$ mass dark matter. We show that both cases can be realized by appropriate choice of two Higgs doublet sector. First, we show that WIMP with about $10 \mathrm{GeV}$ mass can mostly annihilate into tau leptons through the second Higgs boson exchange process with so-called Type$\mathrm{X}$ THDM and could account for the GC excess. ${ }^{16}$ Next, we consider cases where WIMP with the mass about $30-40 \mathrm{GeV}$ mostly annihilate into $b \bar{b}$. With the second Higgs boson with the mass about $60-70 \mathrm{GeV}$ in the so-called Type-II THDM, the resultant annihilation cross section is suitable to explain the cosmic dark matter relic abundance as well as the GC excess. ${ }^{17}$ However, such a light extra Higgs boson in (non-supersymmetric) Type-II THDM is very severely constrained by the search result at the LHC. ${ }^{18}$ This difficulty can be avoided with the Type-Y THDM. ${ }^{19}$ Moreover, we find that signals due to this new light Higgs boson mediator can be observed at the international linear collider (ILC). ${ }^{19}$

\section{Two Higgs doublet portal scalar dark matter}

We introduce a real SM gauge singlet scalar $\phi$ as the dark matter candidate, along with the $Z_{2}$ parity by which the stability of the DM particle is guaranteed. We 
extend the Higgs sector to be two Higgs doublet model with a softly broken $Z_{2}$ parity in order to avoid large flavor changing neutral current. The scalar potential for the two Higgs doublets $\left(\Phi_{1}\right.$ and $\left.\Phi_{2}\right)$ with the soft breaking $Z_{2}$ parity and the scalar DM $\phi$ is given by

$$
\begin{aligned}
V= & -\mu_{1}^{2}\left|\Phi_{1}\right|^{2}-\mu_{2}^{2}\left|\Phi_{2}\right|^{2}-\left(\mu_{12}^{2} \Phi_{1}^{\dagger} \Phi_{2}+\text { H.c. }\right) \\
& +\lambda_{1}\left|\Phi_{1}\right|^{4}+\lambda_{2}\left|\Phi_{2}\right|^{4}+\lambda_{3}\left|\Phi_{1}\right|^{2}\left|\Phi_{2}\right|^{2}+\lambda_{4}\left|\Phi_{1}^{\dagger} \Phi_{2}\right|^{2}+\left\{\frac{\lambda_{5}}{2}\left(\Phi_{1}^{\dagger} \Phi_{2}\right)^{2}+\text { H.c. }\right\} \\
& +\frac{1}{2} \mu_{\phi}^{2} \phi^{2}+\lambda_{\eta} \phi^{4}+\left(\sigma_{1}\left|\Phi_{1}\right|^{2}+\sigma_{2}\left|\Phi_{2}\right|^{2}\right) \frac{\phi^{2}}{2} .
\end{aligned}
$$

Two electrically neutral $C P$-even Higgs bosons, the SM-like Higgs boson $h$ and the other boson $H$, appears at the electroweak symmetry breaking vacuum. We impose the condition $\sin (\beta-\alpha)=1$ with $\tan \beta=v_{2} / v_{1}$ and the mixing angle of those Higgs fields $\alpha$, then $h$ is indeed the SM-like. For simplicity, we assume that the charged and $C P$-odd Higgs bosons are heavy enough to be consistent with their current experimental bound and to decouple them from our analysis of the dark matter physics. Thus, the relevant interaction operators between $\phi$ and Higgs bosons ( $h$ or $H)$ are

$$
\mathcal{O} \supset v H \phi^{2}, \quad v h \phi^{2}
$$

with $v=246 \mathrm{GeV}$ being the vacuum expectation value of Higgs fields. Four ways of this $Z_{2}$ parity assignment in the Higgs sector are referred to as Type-I, II, X and Y, as shown in Table 1. We only consider later three cases, whose Yukawa couplings are given by

$$
\begin{aligned}
& \mathcal{L}_{I I}=-y_{\ell_{i}} \bar{L}^{i} \Phi_{1} \ell_{R}^{i}-y_{u_{i}} \bar{Q}^{i} \tilde{\Phi}_{2} u_{R}^{i}-y_{d_{i}} \bar{Q}^{i} \Phi_{1} d_{R}^{i}+\text { H.c. } \\
& \mathcal{L}_{X}=-y_{\ell_{i}} \bar{L}^{i} \Phi_{1} \ell_{R}^{i}-y_{u_{i}} \bar{Q}^{i} \tilde{\Phi}_{2} u_{R}^{i}-y_{d_{i}} \bar{Q}^{i} \Phi_{2} d_{R}^{i}+\text { H.c. } \\
& \mathcal{L}_{Y}=-y_{\ell_{i}} \bar{L}^{i} \Phi_{2} \ell_{R}^{i}-y_{u_{i}} \bar{Q}^{i} \tilde{\Phi}_{2} u_{R}^{i}-y_{d_{i}} \bar{Q}^{i} \Phi_{1} d_{R}^{i}+\text { H.c. }
\end{aligned}
$$

respectively. Here $Q^{i}\left(L^{i}\right)$ is the ordinary left-handed $S U(2)$ doublet quark (lepton) of the $i$ th generation, and $u_{R}^{i}, d_{R}^{i}$ and $\ell_{R}^{i}$ are the right-handed $S U(2)$ singlet upand down-type quarks and charged leptons, respectively.

We evaluate

- The invisible decay branching ratio of $h$ into a pair of $\phi$ through the interactions in Eq. (2)

Table 1. The assignment of (softly broken) $Z_{2}$ parity for each Type of Yukawa couplings.

\begin{tabular}{|l||c|c|c|c|c|c|}
\hline Type & Others & $\Phi_{1}$ & $\Phi_{2}$ & $u_{R}$ & $d_{R}$ & $\ell_{R}$ \\
\hline \hline Type-I & + & + & - & - & - & - \\
\hline Type-II & + & + & - & - & + & + \\
\hline Type-X & + & + & - & - & - & + \\
\hline Type-Y & + & + & - & - & + & - \\
\hline
\end{tabular}


- The thermal relic abundance of the real scalar DM

- The present annihilation cross section $(\sigma v)_{0}$ of the DM particle

and impose the current bound and future expected sensitivities of DM direct search experiments on those model parameter space.

\subsection{With Type-X}

In the Type-X THDM, $\Phi_{1}$ couples with only leptons. Thus, the non-SM-like Higgs boson $H$, which is mostly comes from $\Phi_{1}$, has large couplings to leptons. A pair of scalar dark matter particles with mass $m_{\phi} \simeq 10 \mathrm{GeV}$ dominantly annihilates into $\tau \bar{\tau}$ through the $s$-channel $H$ exchange. The cross section is enhanced when $m_{H} \sim 2 m_{\phi}$.

Figure 1 shows results for a benchmark point. Since $h$ may have a large invisible decay in some parameter region with a particular particle mass spectrum, ${ }^{20}$ we also have taken this constraint into account. The thick blue line corresponds to the thermal relic abundance of the scalar dark matter $\Omega h^{2}=0.1$, while the parameters between the two dashed red lines provide the annihilation cross section, $2.81 \times$ $10^{-26} \leq(\sigma v)_{0} \leq 3.99 \times 10^{-26} \mathrm{~cm}^{3} / \mathrm{s}$. The shaded regions are excluded by the direct dark matter search at the XENON100 (2012), ${ }^{21}$ and the expected sensitivity by the XENON1T experiment ${ }^{22}$ is depicted by two dotted lines. Near the resonance pole $m_{\phi} \simeq m_{H} / 2$, the thermal relic abundance and a sufficient present annihilation cross section into a tau pair to account for GC excess are simultaneously satisfied for $\sigma_{1} \simeq 0.03, m_{H} \simeq 26 \mathrm{GeV}$. This region is found to be close to the sensitivity of the direct dark matter search expected by the XENON1T experiment.

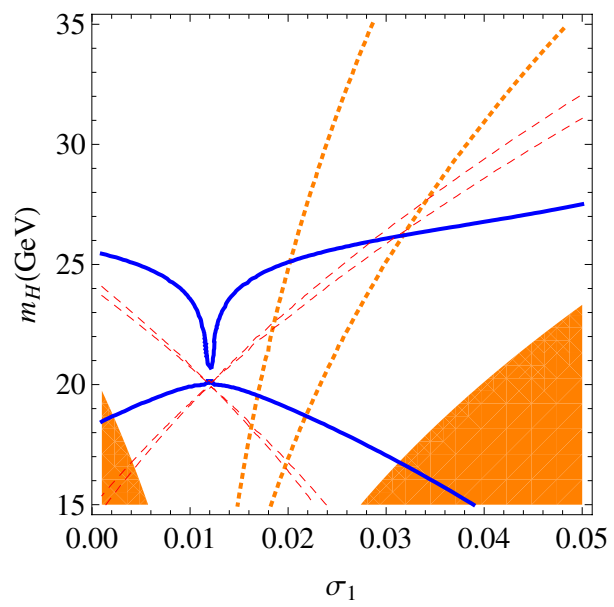

Fig. 1. For explanation of each lines and regions, see the text.

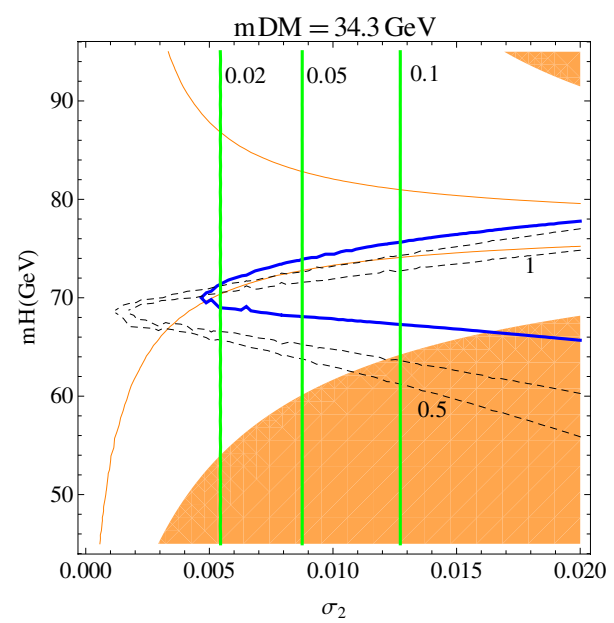

Fig. 2. For explanation of each lines and regions, see the text. 


\subsection{With Type-II}

A pair of scalar dark matter particles with mass $m_{\phi}=31-40 \mathrm{GeV}$ dominantly annihilates into $b \bar{b}$ through the $s$-channel exchange of the Higgs bosons $(h$ and $H$ ). The cross section is enhanced by $H$ exchange when $m_{H} \sim 2 m_{\phi}$. As in the case with Type-X two Higgs doublet fields, there is a parameter region where both the gamma ray excess and the observed relic abundance can be explained simultaneously. ${ }^{17}$ However, as stated in introduction, such a light Higgs boson now confronts with extra Higgs bosons search at the LHC. ${ }^{18}$

\subsection{With Type-Y}

With Type-Y THDM, $\phi$ mainly annihilates into a $b \bar{b}$ pair through the $H$ exchange but $H$ has much smaller couplings with tau lepton compared to those in Type-II Yukawa couplings and less constrained by the LHC. The cross section is enhanced at the $H$ resonance pole when $m_{H} \sim 2 m_{\phi}$. We evaluate the cross section as a function of the coupling $\sigma_{2}$ and the non-SM-like Higgs boson mass $m_{H}$. Figure 2 shows the results for $m_{\phi} \simeq 34 \mathrm{GeV}$, which corresponds to $m_{H}=70 \mathrm{GeV}$. We have fixed $\tan \beta=20$.

Along the thick blue line, the observed DM relic density $\Omega h^{2}=0.1$ is reproduced, while dashed lines show the present DM annihilation cross sections $(\sigma v)_{0}$ and its numbers are shown in units of $10^{-26} \mathrm{~cm}^{3} / \mathrm{s}$. The shaded regions are excluded by the LUX (2014) experiment, ${ }^{23}$ and the expected sensitivity of the XENON1T experiment $^{22}$ is depicted by two thin lines.

As stated above, the SM-like Higgs boson $h$ can decay into a pair of scalar DM particles through the interactions in Eq. (2) which is observed as its invisible decay. Figure 2 also shows the branching ratio of this invisible decay $\mathrm{BR}(h \rightarrow \phi \phi)=0.02$, 0.05 , and 0.1 .

This extra Higgs boson may be discovered at the ILC. The signal process is $e^{-} e^{+} \rightarrow b \bar{b} H \rightarrow b \bar{b} b \bar{b}$. We show the expected numbers of $4 b$ event at the ILC for the center of energy $\sqrt{s}=250 \mathrm{GeV}$ and the luminosity $\mathcal{L}=500 \mathrm{fb}^{-1}$ at Fig. 3 . The
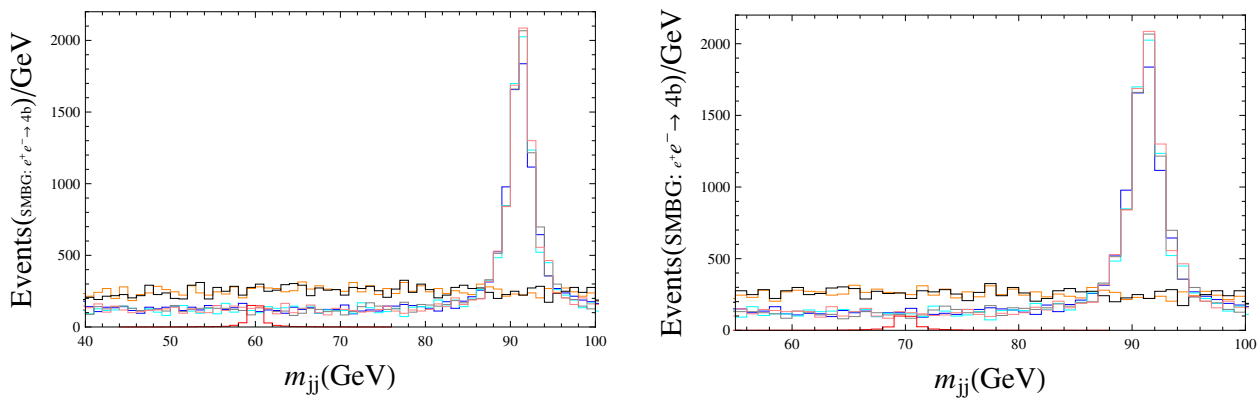

Fig. 3. The expected numbers of $4 b$ events generated by various processes at the ILC for $m_{H}=60$ $\mathrm{GeV}$ (left) and $70 \mathrm{GeV}$ (right), respectively. $m_{j j}$ denotes the invarinat mass. The $H$ mediation contributions are shown with red color in the histogram. 


\section{A. Das, N. Okada \&3 O. Seto}

mediator $H$ exchange contribution are shown with red color in the histogram. For instance, with this number of events of $m_{H}=70 \mathrm{GeV}$, this significance is about $3.4 \sigma$.

\section{Summary}

Motivated by the gamma-ray excess from the Galactic Center and its interpretation as annihilating dark matter particles, we have examined a Higgs portal DM scenarios with two Higgs doublet models. We have identified the model parameter region that can explain the gamma-ray excess and satisfies the phenomenological constraints on the relic dark matter abundance and elastic scattering cross section of the DM particle off nuclei, as well as the invisible decay rate of the SM-like Higgs boson into a pair of DM particles. Most of the identified parameter region can be tested by future direct dark matter detection experiments. In addition, the search for the invisible decay process of the SM-like Higgs boson and the non-SM-like Higgs boson at future collider experiments is complementary to direct DM searches.

\section{Acknowledgments}

This work is supported in part by the DOE Grant No. DE-SC0013680 (N.O) and by the Grant-in-Aid for Scientific Research No. 26400243 and No. 26105514 from the Ministry of Education, Culture, Sports, Science and Technology in Japan (O.S).

\section{References}

1. L. Goodenough and D. Hooper, arXiv:0910.2998 [hep-ph].

2. D. Hooper and L. Goodenough, Phys. Lett. B 697, 412 (2011).

3. D. Hooper and T. Linden, Phys. Rev. D 84, 123005 (2011).

4. K. N. Abazajian and M. Kaplinghat, Phys. Rev. D 86, 083511 (2012).

5. C. Gordon and O. Macias, Phys. Rev. D 88, 083521 (2013) [Phys. Rev. D 89, 049901 (2014)].

6. K. N. Abazajian, N. Canac, S. Horiuchi and M. Kaplinghat, Phys. Rev. D 90, 023526 (2014).

7. F. Calore, I. Cholis and C. Weniger, JCAP 1503, 038 (2015).

8. T. Daylan, D. P. Finkbeiner, D. Hooper, T. Linden, S. K. N. Portillo, N. L. Rodd and T. R. Slatyer, arXiv:1402.6703 [astro-ph.HE].

9. T. Lacroix, C. Boehm and J. Silk, Phys. Rev. D 90, 043508 (2014).

10. M. Ackermann et al. [Fermi-LAT Collaboration], Phys. Rev. Lett. 115231301 (2015).

11. J. McDonald, Phys. Rev. D 50, 3637 (1994).

12. C. P. Burgess, M. Pospelov and T. ter Veldhuis, Nucl. Phys. B 619, 709 (2001).

13. T. Kikuchi and N. Okada, Phys. Lett. B 665, 186 (2008).

14. S. Kanemura, S. Matsumoto, T. Nabeshima and N. Okada, Phys. Rev. D 82, 055026 (2010).

15. A. De Simone, G. F. Giudice and A. Strumia, JHEP 1406, 081 (2014).

16. N. Okada and O. Seto, Phys. Rev. D 89, 043525 (2014).

17. N. Okada and O. Seto, Phys. Rev. D 90, 083523 (2014).

18. G. Aad et al. [ATLAS Collaboration], JHEP 1411, 056 (2014). 
19. A. Das, N. Okada and O. Seto, in preparaion.

20. M. Aoki, S. Kanemura and O. Seto, Phys. Lett. B 685, 313 (2010).

21. E. Aprile et al. [XENON100 Collaboration], Phys. Rev. Lett. 109, 181301 (2012).

22. E. Aprile [XENON1T Collaboration], Springer Proc. Phys. 148, 93 (2013).

23. D. S. Akerib et al. [LUX Collaboration], Phys. Rev. Lett. 112, 091303 (2014). 